\title{
MxA Gene Expression after Live Virus Vaccination: A Sensitive Marker for Endogenous Type I Interferon
}

\author{
A. Roers, H. K. Hochkeppel, M. A. Horisberger, \\ A. Hovanessian, and O. Haller
}

\author{
Abteilung Virologie, Institut für Medizinische Mikrobiologie und Hygiene \\ der Universität Freiburg. Germany; Pharmaceuticals Research \\ Laboratories, CIBA-GEIGY, Lid., Basel, Switzerland; Unité de Virologie \\ et Immunologie Cellulaire, Institut Pasteur, Paris, France
}

\begin{abstract}
MxA gene expression is known to be regulated tightly and exclusively by type I interferons (IFNs). The kinetics of MxA gene expression was analyzed in peripheral blood mononuclear cells from 11 healthy volunteers vaccinated with the 17-D strain of yellow fever virus. A reliable induction of MxA RNA and MxA protein was found in the absence of easily detectable serum IFN activity. Thus, steady-state MxA RNA levels were elevated 8- to 30-fold above prevaccination levels on day 5 after vaccination. The average increase of $\mathrm{MxA}$ protein was $\sim 50$-fold. In contrast, no induction of MxA RNA or MxA protein was detectable in 3 similarly vaccinated controls who were immune because of previous vaccinations. The IFN marker $2^{\prime}$-5'-oligoadenylate (2-5A) synthetase known to react to both type I and type II IFNs showed a similar response but did not differentiate equally well between nonimmune and immune vaccinees. $\beta_{2}$-microglobulin and neopterin reacted poorly, remaining at low levels within the normal range. These results demonstrate that MxA gene expression is a good marker for detecting minute quantities of biologically active type I IFN during viral infections.
\end{abstract}

Interferons (IFNs) are produced in response to viral infection and contribute to host defense by establishing an antiviral state in target cells [1]. Viruses induce predominantly two classes of IFNs, namely IFN- $\alpha$ and IFN- $\beta$, collectively called type I IFNs [2]. It is not always possible, however, to detect circulating type I IFNs in the serum of patients most likely because type I IFNs are produced early in infection and may no longer be present in later serum samples or may not be formed in detectable quantities. For example, serum IFN is not usually found in patients with acute viral hepatitis $[3,4]$, although IFN-induced changes occur in the liver and are suggestive of local IFN production and action [5]. Therefore, more reliable alternatives to direct serum IFN measurements are needed.

Several assays have been developed that are based on the capacity of IFNs to induce expression of IFN-responsive genes in peripheral blood mononuclear cells (PBMC). Increased levels of an IFN-induced gene product indicate that the cells have been exposed to IFN in vivo and are respond-

Received 17 June 1993; revised 23 November 1993.

Presented in part: annual meeting. International Society for Interferon Research. Nice, France, November 1991 (J Interferon Res 1991;1 1:254); IX International Congress of Virology, Glasgow, United Kingdom, August 1993 (abstract 1948a)

All study participants gave informed consent.

Financial support: Deutsche Forschungsgemeinschaft and Forschungsschwerpunktprogramm Baden-Württemberg.

Reprints or correspondence: Dr. Otto Haller, Dept. of Virology, University of Freiburg. Hermann-Herder-Strasse 11, P.O. Box 820, D-79104 Freiburg. Germany.

The Journal of Infectious Diseases 1994;169:807-13 (c) 1994 by The University of Chicago. All rights reserved. $0022-1899 / 94 / 6904-0015 \$ 01.00$ ing to it. One of the most widely used assays measures the induction of an intracellular enzyme, 2', 5'-oligoadenylate (2$5 A)$ synthetase [6-8]. This enzyme assay is considered to be more sensitive than IFN measurements in serum [6] and has the additional advantage that elevated enzyme activities remain detectable long after IFN has disappeared from the blood [9]. Other tests measure p68 protein kinase, indoleamine 2,3-dioxygenase or guanylate binding proteins in PBMC [10-12], or determine secreted products such as $\beta_{2}-$ microglobulin in serum [13] or neopterin in urine [14]. These tests indicate elevated activities not only of type I IFN but also of type II $(\gamma)$ IFN that is produced by $T$ lymphocytes in the course of an immune reaction [2].

An exception is the human MxA protein, which qualifies as a specific biochemical marker for the action of IFN- $\alpha$ and IFN- $\beta[15,16]$. The human MxA protein is a GTPase with antiviral activity against orthomyxoviruses and certain other negative-strand RNA viruses [17-20]. MxA gene expression in human cells is tightly and specifically regulated by type I IFNs $[15,16]$. The specificity and dose response of MxA expression in cell culture have been well established $[15,16$, $21,22]$. The MxA gene in PBMC does not respond to tumor necrosis factor or to a number of interleukins and growth factors and shows only a minimal response to IFN- $\gamma$, which seems to act indirectly via IFN- $\alpha / \beta[15,16]$. Immunoblot assays have been used to monitor MxA protein expression during IFN- $\alpha$ treatment of cancer patients $[23,24]$ or to demonstrate the presence of MxA protein in PBMC from patients with diseases in which acid-labile serum IFN- $\alpha$ seems to play a role $[25,26]$. However, the kinetics of MxA gene expression in vivo during an acute viral infection has not previously been established. 
Here, we analyzed MxA RNA and protein expression in the course of a sclf-limiting viral infection and compared the usefulness of MxA as a marker for endogenous type I IFN with that of various classic IFN markers.

\section{Materials and Methods}

Vaccination and determination of antibody response. The 17D strain of yellow fever virus (YFV; Robert Koch Institut, Berlin) was used for standard subcutaneous vaccination. Antibodies to YFV were determined by hemagglutination inhibition at the Institut für Tropenmedizin (Hamburg, Germany). Three persons known to have been previously vaccinated served as immune controls.

Isolation of plasma and PBMC. Venous blood samples containing EDTA as anticoagulant were processed within $1 \mathrm{~h}$ of drawing. Plasma was obtained by mild centrifugation and was frozen at $-70^{\circ} \mathrm{C}$. PBMC isolated by Ficoll-Paque gradient centrifugation (Pharmacia, Freiburg) were washed twice in PBS and dispensed into aliquots of 2.5 and $5 \times 10^{6}$ cells. The cells were then pelleted in Eppendorf tubes (Eppendorf-NethelerHinz, Hamburg), shock-frozen in dry ice/ethanol, and stored at $-70^{\circ} \mathrm{C}$.

IFN assays. Serum type I IFN activity was determined in a bioassay as described by Rubinstein et al. [27] using MadinDarby bovine kidney cells and vesicular stomatitis virus. The assay was calibrated against National Institutes of Health (Bethesda, MD) IFN- $\alpha$ standard G023-901-527. IFN- $\alpha 2$ a concentrations in plasma samples were determined in the laboratory of U. Elsässer (Institut für Immunbiologie, Freiburg), by EIA (Hoffmann-La Roche, Grenzach-Wyhlen, Germany).

The MxR assay was done exactly as described [28]. Vero $\mathrm{Mx}$ 13 cells (gift of C. Weissmann, Institute of Molecular Biology I, University of Zürich, Switzerland) were grown to confluency in Dulbecco's MEM supplemented by $10 \%$ heat-inactivated fetal bovine serum and $1 \mathrm{mg} / \mathrm{mL} \mathrm{G418}$. The cells were then seeded into six-well plates (Costar/Tecnomara. Fernwald, Germany) at a density of $10^{6}$ cells per well $(3.5-\mathrm{cm}$ diameter). After $24 \mathrm{~h}$, the cells were washed with PBS, and $1 \mathrm{~mL}$ of medium containing $5 \%$ of the serum to be assayed was added. Aliquots of this medium were frozen immediately after addition to the cells and after a 24-h incubation period. Human growth hormone (hGH) content of the aliquots was measured by RIA (Cis/Bio International, Dreieich, Germany). The amount of hGH that had been produced within $24 \mathrm{~h}$ was calculated by subtracting the hGH concentration of the first aliquot from that of the second. IFN standards (IFN- $\alpha 2 \mathrm{a}$, Roferon; Hoffmann-La Roche), which were included, allowed the estimation of serum IFN concentrations corresponding to hGH production.

RNA isolation and analysis. Total cellular RNA was isolated from PBMC as described by Chomcynski and Sacchi [29]. Briefly, pellets of $5 \times 10^{6}$ PBMC were resuspended by sharp vortexing in lysis buffer containing guanidinium-thiocyanate. A first phenol-chloroform-isoamyl alcohol extraction was followed by a second extraction, this time using chloroform-isoamyl alcohol only, and precipitation of RNA with 2-propanol. The RNA was then redissolved in lysis buffer, precipitated again, and recovered by centrifugation. Finally, the pellet was washed with $80 \%$ ethanol. Northern blotting was done according to standard procedures [30]. Slot blotting was done as described previously [15]. Briefly, RNA samples were serially twofold diluted in a solution containing $50 \mathrm{mg} / \mathrm{mL}$ yeast tRNA (Boehringer, Mannheim, Germany). The dilution tubes were incubated at $65^{\circ} \mathrm{C}$ for $20 \mathrm{~min}$ after addition of a formaldehydestandard saline citrate solution, followed by shock cooling on ice. The first six dilutions were then blotted on nitrocellulose membranes (Schleicher \& Schuell, Dassel, Germany) using the minifold II apparatus (Schleicher \& Schuell) according to the manufacturer's protocol. The membranes were hybridized to radiolabeled cDNA probes under standard high-stringency conditions. The MxA probe consisted of the complete coding region of the MxA cDNA cut from the plasmid BShuMxC4b by EcoRI digestion [21]. The human $\beta$-actin probe consisted of the complete coding region of the $\beta$-actin cDNA cut from the plasmid pCDVI by BamHI digestion [31]. Both probes were labeled with [ ${ }^{32}$ P]dCTP (Amersham-Buchler, Braunschweig, Germany) using a nick translation kit (Amersham-Buchler).

ELISA for MXA protein. Protein extracts from pellets of 2.5 $\times 10^{6}$ PBMC or whole blood were assayed in a MxA-specific ELISA exactly as described [32].

Measurement of 2-5A synthetase activity. 2-5A synthetase activity was measured in extracts from PBMC as described by Buffet-Janvresse et al. [33].

Assays for $\beta_{2}$-microglobulin and neopterin. $\quad \beta_{2}$-microglobulin and neopterin concentrations in plasma samples were quantitated by commercially available RIAs (Pharmacia, Freiburg, and Henning, Berlin, respectively).

\section{Results}

Yellow fever vaccination. Eleven healthy adults were vaccinated with 17-D YFV vaccine strain to produce a self-limiting viral infection. They were studied for MxA gene expression as a marker for endogenous IFN. One volunteer, who will be called "patient $x$," was monitored more closely than the rest. Clinical signs of infection did not develop, but seroconversion occurred in all cases indicating successful vaccination. Three additional volunteers were vaccinated and served as negative controls. They had been vaccinated before and were already immune to YFV.

Serum IFN. Vaccination of nonimmune persons led to a small increase in serum type I IFN titers that was most pronounced on day 7 after vaccination (table 1). The low IFN activity measured was barely above the detection limit of the bioassay, yet provided good evidence for systemic infection with the attenuated virus. In contrast, no increase in IFN activity was found in the 3 immune controls, indicating absence of viremia due to neutralization of the vaccine. A novel sensitive assay specific for biologically active human type I IFN, the MxR assay, has been described by Lleonart et al. [28]. This assay makes use of the IFN-responsive murine Mxl promoter, which is similar to the human $\mathrm{MxA}$ promoter [34]. It discriminates against IFN- $\gamma$ and other cytokines and 
Table 1. Serum type I interferon (units/mL) after yellow fever virus vaccination.

\begin{tabular}{|c|c|c|c|c|c|c|c|c|c|c|c|c|c|}
\hline \multirow{2}{*}{$\begin{array}{l}\text { Days after } \\
\text { vaccination }\end{array}$} & \multicolumn{10}{|c|}{ Healthy nonimmune persons } & \multicolumn{3}{|c|}{$\begin{array}{l}\text { Immune } \\
\text { controls }\end{array}$} \\
\hline & 1 & 2 & 3 & 4 & 5 & 6 & 7 & 8 & 9 & 10 & 11 & 12 & 13 \\
\hline 0 & $<6$ & $<3$ & $<18$ & $<6$ & $<6$ & $<6$ & $<6$ & $<6$ & $<3$ & $<9$ & $<6$ & 6 & $<6$ \\
\hline 3 & $<6$ & $<3$ & $<18$ & 6 & $<6$ & ND & ND & ND & ND & ND & ND & 6 & $<6$ \\
\hline 5 & 15 & 6 & $<18$ & 9 & $<6$ & $<6$ & ND & ND & ND & ND & ND & 6 & $<6$ \\
\hline 7 & 9 & 6 & $<18$ & 18 & 9 & 18 & 9 & 9 & 9 & 18 & $<6$ & 6 & $<6$ \\
\hline 11 & $<6$ & $<3$ & $<18$ & $<6$ & $<6$ & $<6$ & ND & ND & ND & ND & $<6$ & 6 & $<6$ \\
\hline
\end{tabular}

NOTE. Serum samples were obtained at times after vaccination indicated and assayed for interferon activity in plaque-reduction assay as described in Material and Methods. ND, not done.

thus should provide an excellent test system for MxA-inducing IFN activities in human sera. Indeed, using this assay, circulating type I IFN could be demonstrated in some of the serum samples of patient $x$. The detectable IFN concentrations were low, again barely exceeding the detection limits of the assay ( $\sim 2-3$ units/mL; data not shown). An EIA specific for IFN- $\alpha 2$ a failed to detect IFN in any of the samples analyzed, indicating that this IFN subtype, if present, was below the detection limit of 0.5 units/mL (data not shown).

Kinetics of MXA RNA expression in vivo. To determine the in vivo kinetics of MxA gene expression, we analyzed steady-state levels of MxA RNA in PBMC obtained from patient $x$ by the slot-blotting technique (figure $1 \mathrm{~A}$ ). Low basal levels of MxA transcripts were detectable in the prevaccination sample, as described previously [21]. The intensity of the MxA signal did not change significantly during the first $24 \mathrm{~h}$ after vaccination. An increase in MxA signal intensity was clearly visible $36 \mathrm{~h}$ after vaccination. The signal remained enhanced between days 2 and 7. Steady-state MxA RNA levels were highest on days 5 and 7 when they were increased $\sim 30$-fold above day 0 levels. The signal was back to normal on day 11. Likewise, the MxA gene was regularly induced in the other 6 nonimmune individuals in which a pre- and postvaccination sample was analyzed for MxA RNA, although the extent of induction varied between 8and 32-fold. Figure $1 B$ shows results for 3 subjects. Northern blot analysis showed that the MxA RNA was of the proper size (data not shown). No induction of MxA RNA was found in the immune controls (figure IB).

Kinetics of $M x A$ protein expression in vivo. Next, intracellular MxA protein concentrations were determined in aliquots of the same samples using a recently established MxA ELISA [32]. Figure 2A shows the results obtained with PBMC of patient $\mathrm{x}$. MxA protein concentrations were low before vaccination and remained low for the first 24-30 h after vaccination. They subsequently increased 70 -fold from 20 to $1400 \mathrm{ng} / 2.5 \times 10^{6}$ cells. This maximum was reached on day 7 after vaccination. Thereafter, MxA concentrations decreased slowly and were still elevated threefold on day 19 . The in vivo half-life of $\mathrm{MxA}$ protein was estimated to be $\sim 4$ days as judged from the decrease of the slope after day 11 when MxA gene expression had virtually stopped. Furthermore, ELISA quantitation of MxA protein was done in whole blood samples from 10 additional nonimmune volunteers (figure 2B). A marked increase in MxA protein concentrations, 50-fold on average, was reproducible in all of them. In contrast, MxA protein content remained at basal levels in all 3 immune subjects (figure 2C).

Comparison of different markers for IFN action. Several IFN-inducible genes are activated by type I and type II IFNs. Their products have previously been used as markers for IFN $[6,8,12,22,23]$. We therefore determined in parallel the kinetics of the three most widely used IFN markers. Aliquots of blood samples from patient $x$ were analyzed for 2-5A synthetase activity, $\beta_{2}$-microglobulin, and neopterin (figures 3 , 4). There was a rapid increase in 2-5A synthetase activity in PBMC ( $\geqslant 15$-fold) during the first 7 days after vaccination. The increase and decrease of enzyme levels paralleled the observed changes in MxA protein concentrations (figure $3 A$ ). Likewise, an increase in enzyme activity was found in most of the 10 other nonimmune subjects (figure 3B). However, there was considerable variability. For example, the 2-5A synthetase activity remained at basal levels in 1 subject, although MxA protein concentrations increased from 5.0 to $421.3 \mathrm{ng} / \mathrm{mL}$ of blood in aliquots of blood samples obtained on days 0 and 7, respectively. Moreover, 2 of the 3 immune controls also reacted and showed a 2-5A synthetase induction similar to that of nonimmune persons (figure $3 \mathrm{C}$ ).

Plasma concentrations of $\beta_{2}$-microglobulin rose temporarily after patient $x$ was vaccinated. They exceeded the normal upper limit of $3.0 \mathrm{mg} / \mathrm{L}$ on day 7 , reaching $3.5 \mathrm{mg} / \mathrm{L}$, but returned to low levels by day 11 (figure 4A). The plasma levels of neopterin behaved similarly, increasing about twofold, with a peak on day 7 just above the normal upper limit of $2.5 \mathrm{ng} / \mathrm{mL}$ (figure $4 \mathrm{~A}$ ). Serum $\beta_{2}$-microglobulin also increased in all nonimmune subjects tested (figure $4 B$ ). With concentrations remaining within the normal range in most instances, this increase was modest but significant. No change was observed in immune controls (figure 4C). Neopterin levels were not investigated further. 


\section{Discussion}

We compared the usefulness of MxA as a marker for endogenous type I IFN with various classic IFN markers, such as 2-5A synthetase, $\beta_{2}$-microglobulin, and neopterin, monitoring them in parallel during a controlled and self-limiting viral infection in otherwise healthy adults. Inoculation of attenuated viruses, such as measles or YFV vaccines, usually leads to systemic infection and activation of the IFN system $[35,36]$. We chose the YFV vaccine, since it is innocuous yet causes a viremia that occurs uniformly between days 3 and 9

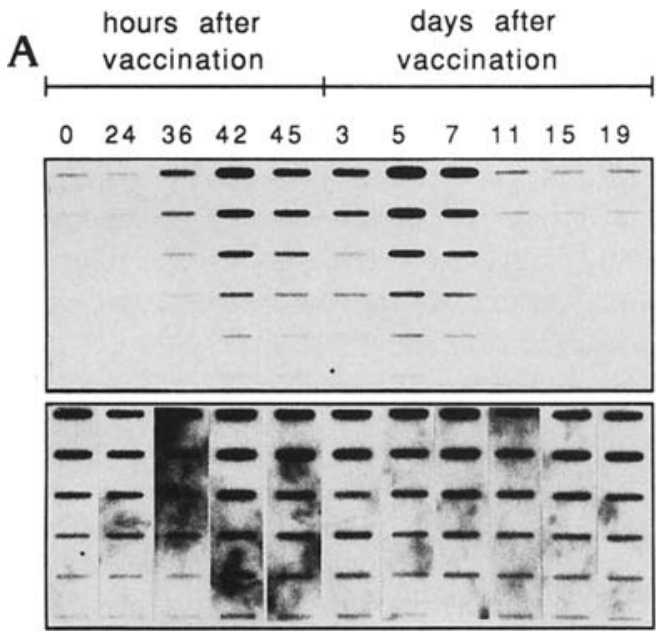

$\mathrm{M} \times \mathrm{A}$
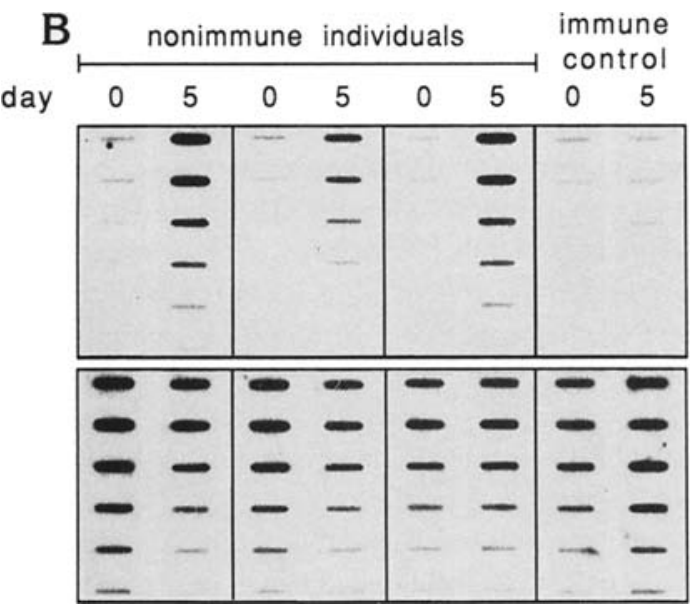

$\mathrm{M} \times \mathrm{A}$

Actin

Figure 1. Kinetics of MxA RNA expression in peripheral blood mononuclear cells (PBMC) during infection (A) in patient $x$, and (B) in 3 additional nonimmune subjects, and an immune control (B). Blood was taken at times after vaccination indicated; PBMC were isolated by Ficoll-Paque gradient centrifugation. Total cellular RNA was prepared from PBMC $\left(5 \times 10^{6}\right)$ and serially diluted 2-fold. Dilutions were blotted below each other by slot blotting. Original undiluted RNA solution was blotted on top. Membrane was hybridized under standard high-stringency conditions to radiolabeled MxA cDNA probe [21] or to radiolabeled $\beta$-actin cDNA probe [31].
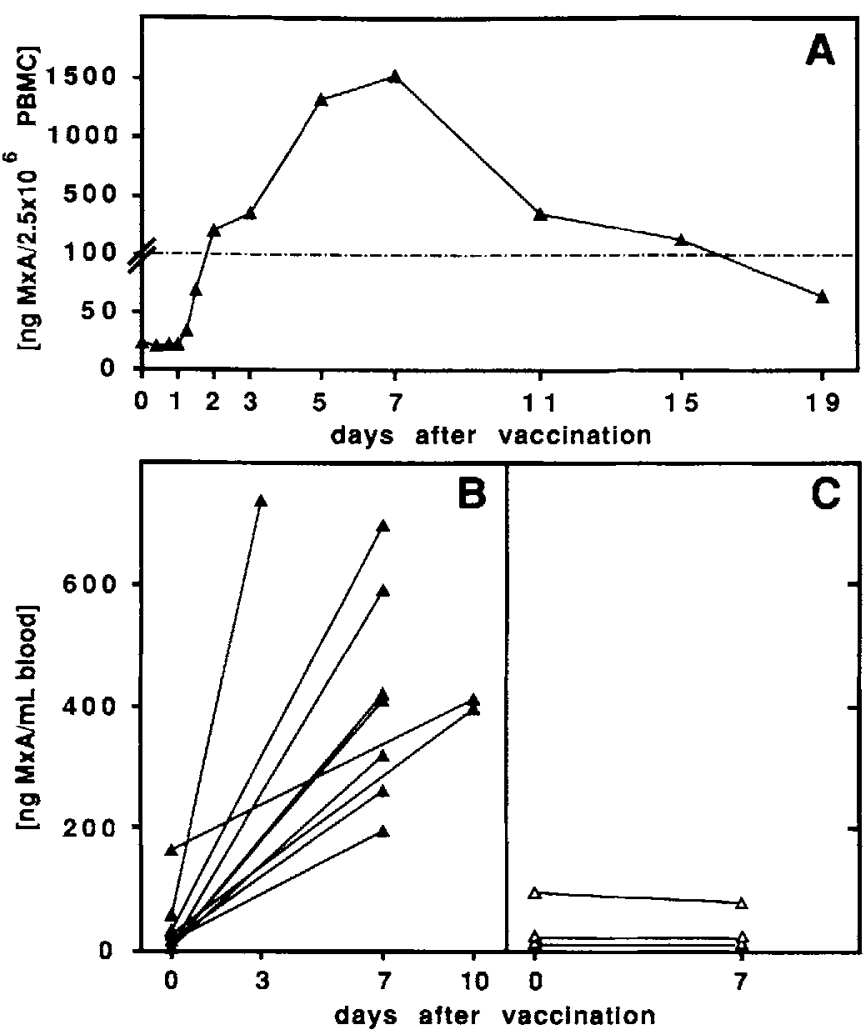

Figure 2. Kinetics of MxA protein expression in peripheral blood mononuclear cells (PBMC) during infection in patient $x$ (A), 10 other nonimmune subjects (B), and 3 immune controls (C). Blood was taken at times after vaccination indicated; MxA protein was quantitated by ELISA [32] in extracts prepared from PBMC $\left(2.5 \times 10^{6}\right)(\mathbf{A})$ or whole blood $(\mathbf{B}, \mathbf{C})$. Coefficient of variation of assay, $<10 \%$. In $\mathbf{B}$ and $\mathbf{C}$, only peak $\mathrm{MxA}$ level after vaccination is shown for each subject.

after vaccination with a peak on day 5 [36]. Most adults are not immune, and infection can easily be demonstrated by an increase in YFV antibodies. In addition, circulating IFN has been found in a majority of vaccinated subjects [36].

IFN markers can be meaningfully compared in vaccinees for three reasons. First, the kinetics of IFN production and action follows the natural course of infection. Second, the endogenous IFN released is of natural subtype composition. This contrasts with the somewhat artificial nature of previous studies in which injection of recombinant IFNs was used to assess the dynamics of single IFN markers in vivo $[12,23$, 24]. Third, previously vaccinated subjects were available who resisted reinfection due to specific immunity. They served as appropriate controls. A conventional bioassay showed low levels of circulating type I IFN in most nonimmune vaccinees but not in similarly vaccinated immune controls. In patient $x$, biologically active type I IFN was detected at very low concentrations by the MxR assay in only some of the serum samples obtained [28]. Because of the short halflife of IFN, serum levels are low in many patients with acute 

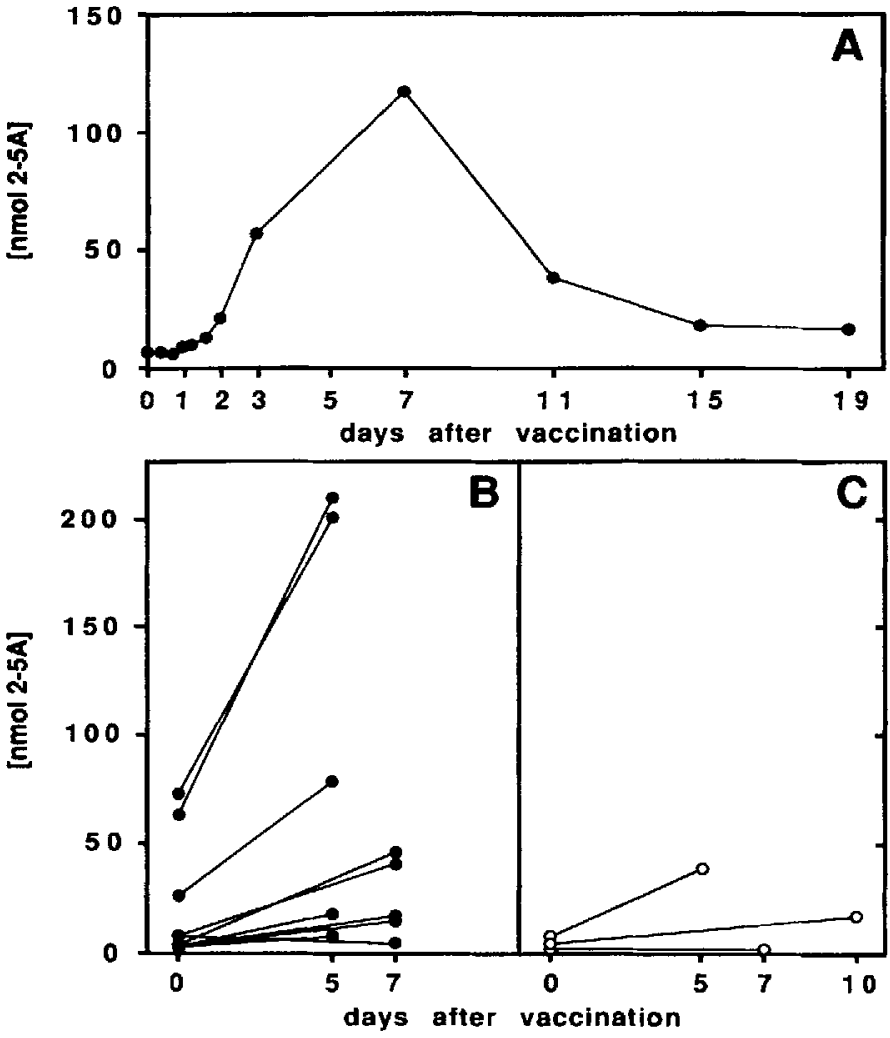

Figure 3. Kinetics of $2^{\prime}-5^{\prime}$-oligoadenylate (2-5A) synthetase activities in peripheral blood mononuclear cells (PBMC) during infection in patient $x(A), 10$ additional nonimmune subjects (B), and $3 \mathrm{immune}$ controls $(\mathbf{C})$. Blood was taken at times after vaccination indicated, and 2-5A synthetase activity was determined in extracts of $2.5 \times 10^{6} \mathrm{PBMC}$. In $\mathbf{A}$, day 5 sample was not available for 2-5A synthetase assay. In $B$ and $C$, only peak activity after vaccination is shown for each subject.

viral diseases $[4,37]$. Fluctuations are caused by continuous production and clearance of IFN.

MxA gene expression could readily be demonstrated in blood cells of all nonimmune volunteers, indicating the cells had been exposed to type I IFN and were responding to it. As expected, no MxA gene activation occurred in immune controls. We have previously shown that enhanced MxA transcription occurs in PBMC within 90 min of a subcutaneous injection of IFN- $\alpha$ into healthy persons [38]. We therefore assume that the increased MxA transcript levels observed after vaccination reflected the appearance of endogenous type I IFN. The remote possibility that viral replication directly activated MxA gene expression is unlikely given the strong induction observed but cannot formally be excluded. We previously showed that the human MxA gene shows almost no primary response to RNA viruses that are otherwise good inducers of IFN [39]. MxA RNA levels rapidly returned to prevaccination levels, reflecting the short half-life of MxA transcripts [38]. Since MxA gene expression shows no refractory phase but can be repeatedly restimulated by IFN [41], these findings indicate that type I IFN production probably ceased between days 7 and 11 after vaccination. Low MxA RNA levels were found in PBMC of all healthy persons studied $[21,22]$ (unpublished data) and can be attributed to the action of small amounts of constitutive IFN [40]

The MxA protein response was even more striking. The in vivo kinetics of $\mathrm{MxA}$ protein accumulation were documented using a newly developed ELISA [32]. This assay allowed quantitation of MxA concentrations in PBMC and showed that the protein rose $\sim 50$-fold above prevaccination levels. More importantly, the decay in MxA protein levels was remarkably slow and MxA protein concentrations were still elevated 19 days after vaccination. MxA protein is known to be quite stable in cells $[22,32,41]$. Its half-life in in vitro IFN-treated PBMC has been estimated to be several days [22, 32]. Ronni et al. [41] recently reported the half-life of MxA protein to be 2.3 days. The situation in vivo is, of course, more complex. During infection, endogenous IFN is produced as a consequence of successive viral replication and may be present in varying concentrations for prolonged periods. By necessity, different blood samples were analyzed at each time point. Under these circumstances, MxA protein in PBMC had a half-life of $\sim 4$ days in vivo as estimated from the decrease in MxA protein concentrations after day 11 (figure $2 \mathrm{~A}$ ) when $\mathrm{MxA}$ gene activity was already back to normal (figure IA). The relatively long half-life makes MxA protein a useful marker for detecting even small amounts of endogenous IFN. If IFN is released continuously for some time, MxA protein will gradually accumulate in PBMC and indicate the presence of biologically active IFN that may otherwise escape direct measurement. If IFN is released over a short period but in sufficient quantity to activate MxA, a single IFN pulse will still be detectable even days later. In both cases, MxA protein will be a good indicator of recent type I IFN activity in vivo. To differentiate between past and present in vivo IFN effects more precisely, MxA mRNA pools in PBMC should be analyzed. Basal levels are constantly low since the MxA gene is silent in the absence of type IIFN $[15,16,21,22]$. As shown here, gene activation in vivo is fast and short lived. Hence, monitoring of MxA mRNA allows detection of sudden changes in IFN activity while measuring MxA protein is more suitable for recording long-term changes.

Among the various IFN markers, 2-5A synthetase has been used the most. Increased enzyme activity has been demonstrated in PBMC of patients with naturally occurring viral diseases [6]. In our experimentally infected individuals, 2-5A synthetase and MxA protein showed similar expression kinetics. The main difference is that the MxA gene in PBMC responds exclusively to type I IFN $[15,16]$, whereas the 2-5A system is activated by both type I and type II IFN [7]. Of interest, there was an induction of 2-5A synthetase activity in 2 of the 3 immune controls. Since no activation of the 

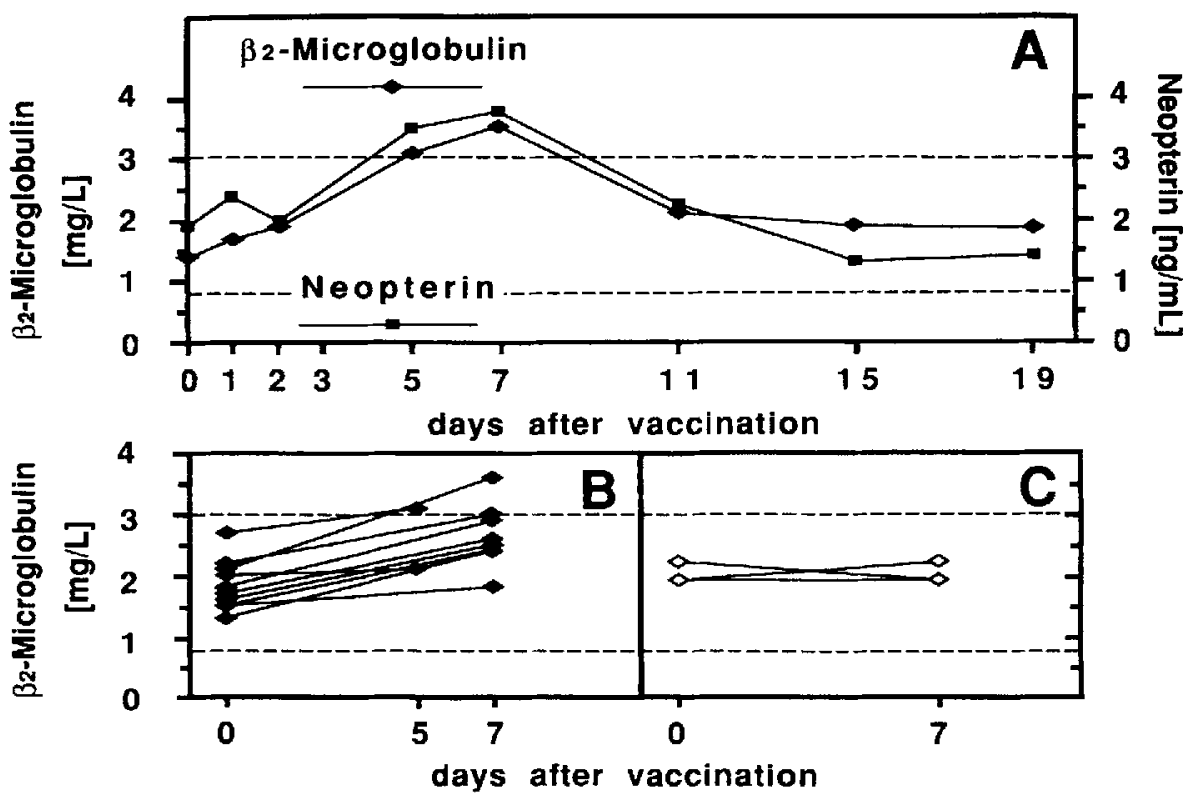

Figure 4. Kinetics of $\beta_{2}$-microglobulin and neopterin serum levels during infection in patient $x(\mathbf{A})$. Coefficients of variation were $<5 \%$ in both assays. Changes in plasma $\beta_{2}$-microglobulin levels are shown in 10 additional nonimmune subjects $(B)$ and in 3 immune controls $(C)$. Dashed lines, normal upper and lower limits of $\beta_{2}$-microglobulin serum concentrations. Increase in $\beta_{2}$-microglobulin serum concentrations on day 7 after vaccination vs. day 0 was significant in the II nonimmune volunteers as estimated by $t$ test for paired samples $(\alpha=.001, f=10)$.
MxA gene was found in these subjects, the induction of the 2-5A system was most likely due to the effect of type II IFN or other cytokines generated during the immune response initiated by revaccination. Therefore, if effects of type I IFNs are to be differentiated from those of IFN- $\gamma$ or other cytokines in vivo, the MxA assay should be used preferentially. Neopterin and $\beta_{2}$-microglobulin are nonspecific $[13,14]$ and reacted poorly in our analysis.

The MxA protein has successfully been used as a clinical marker in patients treated with recombinant IFN- $\alpha[23,24]$ and in patients with diseases in which acid-labile serum IFN$\alpha$ seems to play a role $[42,43]$, such as in those with symptomatic human immunodeficiency virus type 1 infection [25] or systemic lupus erythematosus [26]. The present results indicate that MxA gene expression in PBMC is prominent during an acute viral infection and is a sufficiently sensitive marker to reveal the transient production of very low levels of endogenous IFN that are otherwise barely detectable. Because of their specificity for type I IFN, MxA assays may be most helpful in evaluating diseases with suspected viral etiology and, perhaps, subclinical viral infections or virus carriers. The novel MxA ELISA used here facilitates the handling of large numbers of clinical samples. Monitoring MxA expression could, therefore, become a useful diagnostic tool for detecting even minute quantities of type I IFN.

\section{Acknowledgments}

We thank Charles Weissmann, University of Zürich, for providing Vero-Mx13 cells; Herbert Schmitz, University of Hamburg, for determining anti-yellow fever virus antibodies; Harry Towbin, CIBA GEIGY, Basel, for determining MxA protein in whole blood; Ursula Elsässer, University of Freiburg, for IFN immunoassays; and Jürg Fäh and Peter Staeheli for help and valuable suggestions.

\section{References}

1. Staeheli P. Interferon induced proteins and the antiviral state. Adv Virus Res 1990;38:147-200.

2. Baron S, Coppenhaver DH. Dianzani F, et al. Interferon principles and medical applications. Galveston. TX: University of Texas Medical Branch, 1992.

3. Hill DA, Walsh JH, Purcell RH. Failure to demonstrate circulating interferon during incubation period and acute stage of transfusion associated hepatitis. Proc Soc Exp Biol Med 1971;136:853-6.

4. Pirovino M. Aguet M. Huber M, et al. Absence of detectable serum interferon in acute and chronic viral hepatitis. Hepatology 1986;6:645-9.

5. Thomas HC, Pignatelli $M$, Scully LJ. Viruses and immune reactions in the liver. Scand J Gastroenterol Suppl 1985;114:105-17.

6. Schattner A. Wallach D, Merlin G, Hahn T, Levin S, Revel M. Assay of an interferon-induced enzyme in white blood cells as a diagnostic aid in viral diseases. Lancet 1981;2:497.

7. Witt PL, Spear GT, Helgeson DO, Lindstrom LJ, Smalley RV, Borden EC. Basal and interferon-induced 2',5'-oligoadenylate synthetase in human monocytes, lymphocytes, and peritoneal macrophages. J Interferon Res 1990;10:393-402.

8. Lodemann E, Kornhuber B, Gerein V, von llberg C. (2'-5')oligo(A) synthetase as a monitor of interferon action in juvenile laryngeal papillomatosis. J Interferon Res 1984;4:283-90.

9. Schattner A, Merlin G, Wallach D. Monitoring of interferon therapy by assay of $\left(2^{\prime}-5^{\prime}\right)$ oligo-isoadenylate synthetase in human peripheral white blood cells. J Interferon Res 1981;1:587-94.

10. Buffet-Janvresse C, Vannier JP, Laurent AG, Robert N, Hovanessian AG. Enhanced levels of double-stranded RNA-dependent protein kinase in peripheral blood mononuclear cells of patients with viral infections. J Interferon Res 1986;6:85-96.

11. Carlin JM, Borden EC, Byrne GI. Enhancement of indoleamine 2,3dioxygenase activity in cancer patients receiving interferon- $b_{s e r} . J$ Interferon Res 1989;9:167-73. 
12. Cheng YSE, Becker-Manley MF, Rucker RG, Borden EC. Accumulation of guanylate binding proteins in patients treated with interferons. J Interferon Res 1988;8:385-91

13. Erwin PE. Wibell L. Serum $\beta_{2}$-microglobulin in various disorders. Clin Chim Acta 1973:43: 183-7.

14. Magreiter T, Fuchs D, Hausen A, et al. Neopterin as a new biochemical marker for diagnosis of allograft rejection. Transplantation 1983;36:650-3.

15. Simon A, Fäh J, Haller O, Staeheli P. Interferon-regulated Mx genes are not responsive to interleukin-1, tumor necrosis factor, and other cytokines. J Virol 1991:65:968-71.

16. von Wussow P, Jakschies D. Hochkeppel HK. Fibich C, Penner L, Deicher $\mathrm{H}$. The human intracellular $\mathrm{Mx}$-homologous protein is specifically induced by type I interferons. Eur J Immunol 1990;20: 2015-9.

17. Horisberger MA. Interferon-induced human protein MxA is a GTPase which binds transiently to cellular proteins. J Virol 1992;66:4705-9.

18. Pavlovic J, Zürcher $T$, Haller $O$. Staeheli P. Resistance to influenza virus and vesicular stomatitis virus conferred by expression of human MxA protein. J Virol 1990;64:3370-5.

19. Staeheli P. Pitossi F, Pavlovic J. Mx proteins: GTPases with antiviral activity. Trends Cell Biol 1993;3:268-72.

20. Schnorr JJ, Schneider-Schaulies S, Simon-Jodicke A, Pavlovic J, Horisberger MA, ter Meulen V. MxA-dependent inhibition of measles virus glycoprotein synthesis in a stably transfected human monocytic cell line. J Virol 1993;67:4760-8.

21. Aebi M, Fäh J, Hurt N, et al. cDNA structures and regulation of two interferon induced $\mathrm{Mx}$ proteins. Mol Cell Biol 1989;9:5062-72.

22. Goetschy JF, Zeller H, Content J, Horisberger MA. Regulation of the interferon-inducible IFI-78K gene, the human equivalent of the murine $\mathrm{Mx}$ gene, by interferons, double-stranded RNA, certain cytokines, and viruses. J Virol 1989;63:2616-22.

23. Jakschies D. Hochkeppel HK. Horisberger MA. Deicher H, von Wussow P. Correlation of the antiproliferative effect and the Mx-homologous protein induction by interferon in patients with malignant melanoma. J Invest Dermatol 1990;95:238S-4 IS.

24. Jakschies D. Hochkeppel HK. Horisberger MA. Deicher H, von Wussow P. Emergence and decay of the human $\mathrm{Mx}$ homolog in cancer patients during and after interferon- $\alpha$ therapy. $J$ Biol Response Mod 1990;9:305-12.

25. von Wussow $\mathrm{P}$, Jakschies D. Block $\mathrm{B}$. The interferon-induced $\mathrm{Mx}$-homologous protein in people with symptomatic HIV-1 infection. AIDS 1990;4: 119-24.

26. von Wussow $P$, Jakschies D, Hochkeppel HK, Horisberger MA, Hartung $\mathrm{K}$. Deicher $\mathrm{H}$. Mx homologous protein in mononuclear cells from patients with systemic lupus erythematosus. Arthritis Rheum 1989;32:914-8

27. Rubinstein S, Familletti P, Pestka S. Convenient assay for interferons. J Virol $1981 ; 37: 755-8$
28. Lleonart R, Näf D, Browning $H$, Weissmann C. A novel, quantitative bioassay for type I interferon using a recombinant indicator cell line. Bio/Technology 1990;8:1263-7.

29. Chomcynski P, Sacchi N. Single step method of RNA isolation by acid guanidinium thiocyanate-phenol-chloroform extraction. Anal Biochem 1987;162:156-9.

30. Staeheli P, Danielson P, Haller O, Sutcliffe JG. Transcriptional activation of the mouse $\mathrm{Mx}$ gene by type $\mathrm{I}$ interferon. Mol Cell Biol 1986;6:4470-4.

31. Gunning P, Ponte $P$, Okayama $H$, Engel J, Blau H, Kedes L. Isolation and characterization of full length $\mathrm{cDNA}$ clones for human $\alpha, \beta$ and $\gamma$ actin mRNAs. Mol Cell Biol 1983;3:787-95.

32. Towbin H, Schmitz A. Jakschies D, von Wussow P, Horisberger MA. A whole blood immunoassay for the interferon inducible human $\mathrm{Mx}$ protein. J Interferon Res 1992;12:67.

33. Buffet-Janvresse C, Magard H, Robert N, Hovanessian AG. Assay and the levels of 2-5A synthetase in lymphocytes of patients with viral. bacterial and autoimmune diseases. Annals Immunol (Inst Pasteur) 1983; 134D:247-58.

34. Chang KC, Hansen E, Foroni L, Lida J, Goldspink G. Molecular and functional analysis of the virus- and interferon-inducible MxA promoter. Arch Virol 1991:117:1-15.

35. Petralli JK, Merigan TC, Wilbur JR. Circulating interferon after measles vaccination. N Engl J Med 1965;273:198.

36. Wheelock EF, Sibley WA. Circulating virus, interferon and antibody after vaccination with the 17-D strain of yellow-fever virus. $N$ Engl $J$ Med 1965;273:194-8

37. Parry RP, Parry JV. Interferon assay as a diagnostic test. Lancet 1981:1:506-7.

38. Fäh J, Stacheli P. Haller O. Akkumulation von Mx mRNA als Ma $\beta$ für die biologische Wirksamkeit von Interferon. In: Niederle $N$, von Wussow P. Interferone, präklinische und klinische Befunde. Berlin: Springer-Verlag, 1990:122-36

39. Bazzigher L. Pavlovic J, Haller O, Staeheli P. Mx genes show weaker primary response to virus than other interferon-regulated genes. Virology 1992;186:154-60.

40. Tovey MG. Streuli M, Gresser I, et al. Interferon messenger RNA is produced constitutively in the organs of normal individuals. Proc Natl Acad Sci USA 1987;84:5038-42.

41. Ronni T, Melen K, Malygin A, Julkunen I. Control of IFN-inducible MxA gene expression in human cells. J Immunol 1993;150:171526.

42. Eyster ME, Goedert JJ, Poon MC, Preble OT. Acid-labile alpha interferon, a possible preclinical marker for the acquired immunodeficiency syndrome in hemophilia. $\mathrm{N}$ Engl $\mathbf{J}$ Med 1983;309:583-6.

43. Preble OT, Black RJ, Friedmann RM, Klippel JH, Vilcek J. Systemic lupus erythematosus: presence in human serum of an unusual acid labile leukocyte interferon. Science 1982;216:429-31. 